\title{
A skin in situ immune cell detection kit for the diagnosis and classification of cutaneous lupus erythematosus
}

\author{
Qianwen $\mathrm{Li}^{1 \#}$, Kaili Chen ${ }^{1 \#}$, Yueming Deng ${ }^{1}$, Xiguang Liu ${ }^{2}$, Guozhang $\mathrm{Ma}^{2}$, Jinrong Zeng ${ }^{3}$, Jianyun $\mathrm{Lu}^{3}$, \\ Ming Zhao ${ }^{1}$, Haijing $\mathrm{Wu}^{1}$, Qianjin $\mathrm{Lu}^{1,4,5,6}$ \\ ${ }^{1}$ Department of Dermatology, Second Xiangya Hospital, Central South University, Hunan Key Laboratory of Medical Epigenomics, Changsha, \\ China; ${ }^{2}$ Department of Dermatology, Hei Long Jiang Provincial Hospital, Heilongjiang, China; ${ }^{3}$ Department of Dermatology, Third Xiangya \\ Hospital, Central South University, Hunan Key Laboratory of Medical Epigenomics, Changsha, China; ${ }^{4}$ Institute of Dermatology, Chinese Academy \\ of Medical Sciences and Peking Union Medical College, Nanjing, China; ${ }^{5}$ Key Laboratory of Basic and Translational Research on Immune-Mediated \\ Skin Diseases, Chinese Academy of Medical Sciences, Nanjing, China; ${ }^{6}$ Jiangsu Key Laboratory of Molecular Biology for Skin Diseases and STIs, \\ Nanjing, China \\ Contributions: (I) Conception and design: Q Lu, H Wu, Q Li, K Chen; (II) Administrative support: M Zhao; (III) Provision of study materials \\ or patients: X Liu, G Ma, J Zeng; (IV) Collection and assembly of data: Q Li, K Chen; (V) Data analysis and interpretation: Q Li, K Chen; (VI) \\ Manuscript writing: All authors; (VII) Final approval of manuscript: All authors. \\ "These authors contributed equally to this work. \\ Correspondence to: Qianjin Lu; Haijing Wu. Department of Dermatology, Second Xiangya Hospital, Central South University, Hunan Key Laboratory \\ of Medical Epigenomics, Changsha, China. Email: qianlu5860@pumcderm.cams.cn; chriswu1010@csu.edu.cn.
}

Background: Although lupus can be diagnosed by first impression, medical history, physical examination, pathological analysis and laboratory tests, the accurate classification of cutaneous lupus erythematosus (LE) is still a major challenge in the clinic, which might mislead the selection of treatments and miss the right time for the administration of therapies. The goal of this study was to establish a novel kit to assist with the diagnosis and classification of cutaneous lupus.

Methods: Sixty-five patients from three hospitals were included in this study, including 50 patients with LE and other similar skin diseases. We invited two dermatology specialists to make an accurate diagnosis of the subtypes of lupus based on the patient's clinical features, laboratory examination tests, pathology manifestation analysis, medical treatments and follow-up records. Then, we used their diagnosis results as a standard to which we successively compared the consistency of each step of our diagnosis processes, including impression diagnosis, pathology diagnosis, the combined consideration of the former two diagnostic analyses, and the results of an in situ immune cell detection kit to assist in arriving at a judgement. Results: By Cohen's kappa analysis, we found that the results of the in situ immune cell detection kit had the highest consistency with the diagnoses of the two specialists, both for the diagnosis $(\mathrm{k}=0.921)$ and for the classification of cutaneous lupus $(\mathrm{k}=0.940)$. In addition, this kit enhanced the LE classification accuracy by $36.3 \%$ compared with the diagnostic accuracy of impression diagnosis combined with only pathological analysis.

Conclusions: This skin in situ immune cell detection kit may assist doctors in achieving a higher diagnostic performance and price ratio and enhance their diagnostic efficiency.

Keywords: Cutaneous lupus; classification; in situ immune cell detection kit; diagnostic efficiency

Submitted Feb 28, 2021. Accepted for publication Apr 20, 2021.

doi: $10.21037 /$ atm-21-959

View this article at: http://dx.doi.org/10.21037/atm-21-959 


\section{Introduction}

Lupus is an autoimmune disease with highly heterogeneous symptoms, and one of its noteworthy features is its diverse manifestation of skin lesions; together, these conditions are referred to as cutaneous lupus erythematosus (CLE). According to its speed of onset, CLE is generally classified as chronic cutaneous lupus erythematosus (CCLE), subacute cutaneous lupus erythematosus (SCLE) and acute cutaneous lupus erythematosus (ACLE) (1-3). In the early stages of lupus, the lesions are mostly nonspecific erythema and then they develop different manifestations over time. Owing to the complex and variable skin damage, it is difficult for less experienced dermatologists and young dermatologists to differentiate lupus from other diseases, which show similar lesions such as lichen planus, erythema multiforme, and granuloma annulare. Moreover, pathological analysis of skin biopsy is the gold standard in the diagnosis of cutaneous lupus. However, in addition to the rich working experience and excellent professional skills required for pathologists, in the real world of clinical use, pathologic analysis alone is often insufficient to make the correct classification of lupus. Many cases of lupus classification are only confirmed after double checking the development of the disease and following up the patients (4).

It is critical to classify the subtypes of cutaneous lupus due to the different therapeutic strategies applied for each type. Discoid lupus erythematosus (DLE), the most common type of CCLE, often causes a scarring discoidlike lesion on the face that can linger for a prolonged period, and failure of treatment can result in permanent scarring and hypopigmentation. It is extremely important for dermatologists to diagnose this early because early and effective intervention can promote the resolution of established lesions and prevent scarring in a timely manner (5).

In recent years, there have also been accumulated reports on the association of SCLE with systemic diseases. Up to $15 \%$ of patients with SCLE can be complicated by internal disease. Some SCLE patients may develop serious internal manifestations of Sjögren's, such as pulmonary or neurologic disease, because of the strong correlation between anti-Ro/ SSA autoantibodies and the risk of Sjögren's (6). In addition, it has been reported that up to $30 \%$ of SCLE cases are drug-induced, which is characterized by a higher risk of systemic involvement and an older age at onset $(7,8)$.

Clinically, ACLE is the cutaneous manifestation of systemic lupus erythematosus (SLE). SLE patients usually manifest complicated clinical symptoms such as fever, and multiple systems are involved, including the skin, kidney and joints. Moreover, some SLE patients can be complicated by other autoimmune diseases, such as antiphospholipid syndrome, Raynaud's syndrome, rheumatoid arthritis and dermatomyositis. Compared with DLE and SCLE, once SLE is suspected, a more detailed examination, closer attention to the risk of renal damage, and longer-term follow-up are necessary $(9,10)$. Overall, each type of lupus needs different behavioral interventions and topical and systemic medications (11).

Generally, what we can see from HE staining is the basic structure and the distribution of cells in different shapes, but it is difficult to distinguish different immune cells as they look similar in shape. LE is an autoimmune disease with abnormal lymphocyte proliferation with autoactivated $\mathrm{T}$ and $\mathrm{B}$ cells (12), and thus it is significant that we show a map of immune cell distribution in lupus lesions. As one of the most common techniques in biology and medicine with well-established methods, the utilization of multiple immunohistochemistry (IHC) staining has enabled us to discover specific information on proteins within the tissue and traits that cannot be fully detected by simple HE staining of biopsies of LE skin lesions, especially the morphological features of how a variety of immune cells are distributed. Furthermore, it has been reported that increased B cells are found in sites of chronic inflammation, while in the acute damage response, the rapid migration and proliferation of $\mathrm{T}$ cells dominates (13). The composition and distribution of $\mathrm{T}$ cells and $\mathrm{B}$ cells should be different in different degrees of skin injuries of lupus.

Accurate pathologic diagnosis is essential for the differential diagnosis of cutaneous lesions of lupus. The multiplicity of skin damage and complexity of lupus disease can lead to misdiagnoses between different LE subtypes or other connective tissue diseases, which may lead to improper treatment. Here, we developed a skin in situ immune cell $\left(\mathrm{CD}^{+}\right.$and $\left.\mathrm{CD} 19^{+}\right)$detection kit that could improve the diagnostic efficiency and lead to a more prompt diagnosis of lupus. Based on the urgent need for personalized and precise medicine, both patients and doctors are desperate for a more reliable way to classify LE into specific subtypes. This skin in situ immune cell detection kit may provide a more convenient approach with higher diagnostic performance to enhance diagnostic efficiency for lupus.

We present the study in accordance with the STARD reporting checklist (available at http://dx.doi.org/10.21037/ 
atm-21-959).

\section{Methods}

The study was conducted in accordance with the Declaration of Helsinki (as revised in 2013). All human studies were approved by the ethics committee of the Second Xiangya Hospital of Central South University (No. 201930044). We obtained written informed consent from all subjects. This study was reviewed and approved by the Ethics Committee and carried out in strict accordance with the approved research protocol.

\section{Sample collection and study approval}

Our study group contained 65 patients, all of whom were randomly selected from among patients who were suspected of having lupus based on clinical impressions or pathological opinions and had complete pathology examination records and clinical data (the eligibility criteria and exclusion criteria are shown in Figure 1). These patients were retrospectively recruited from outpatient dermatology clinics and inpatient wards over the past 5 years from the Department of Dermatology, The Second Xiangya Hospital, Central South University; the Department of Dermatology, The Third Xiangya Hospital, Central South University; and the Department of Dermatology, Hei Long Jiang Provincial Hospital, China. All patients were clinically diagnosed and untreated at the time the biopsy was taken. Paraffin sections of 4-6 $\mu \mathrm{m}$ were prepared from the skin biopsies of these patients (sun-exposed areas of lesional skin).

\section{Diagnostic steps}

Based on the clinical features, laboratory tests, pathological analysis, medical treatments and follow-up records that we collected retrospectively, two specialists of the Second Xiangya Hospital made an accurate diagnosis of the subtypes of lupus together. The diagnostic results were used as the gold standard in the analysis of Cohen's kappa. Clinically, dermatologists diagnose these diseases by their "first impression", which is mainly based on the case history, physical examination findings and their medical experience. We collected the first impression diagnosis of doctors from the three hospitals by checking the diagnosis in the pathological examination report.

Pathologists at the three hospitals made pathologic diagnoses depending on the results of HE staining and lupus band tests. The first corrected diagnosis made by doctors is based on the pathologists' report along with their own first impression. The second corrected diagnosis made by doctors is based on the receipt reports from pathologists, the analysis results of the skin in situ Immune Cell Detection Kit, along with their first impression (the detailed diagnostic flow is shown in Figure 1).

\section{Paraffin sections stained by the skin in situ immune cell detection kit}

Pressure cooker antigen repair was performed for 7 minutes in antigen retrieval buffer. The area of skin tissue was covered with blocking buffer for $10 \mathrm{~min}$ at room temperature. Slides were incubated in anti-CD4 working solution for 1 hour, and subsequently with polymer HRP secondary antibodies for $20 \mathrm{~min}$, and finally placed in dying A solution for $20 \mathrm{~min}$. Antibody elution was performed through a high-pressure method. Then, the slides were incubated in anti-CD19 working solution for 1 hour, subsequently with polymer HRP secondary antibodies for $20 \mathrm{~min}$, and finally placed in dying B solution for $20 \mathrm{~min}$. DAPI working solution was applied for $5 \mathrm{~min}$ at room temperature, and then the slices were sealed and examined with a microscope. Antigen retrieval buffer, blocking buffer, anti-CD4 working solution, anti-CD19 working solution, polymer HRP secondary antibodies, dying A solution and dying B solution were all obtained from the skin in situ immune cell detection kit provided by Guangzhou LBP Medicine Science \& Technology Co., Ltd.

\section{Statistical analysis}

The data in this study were analyzed with SPSS 23.0. We used the consistency test (Kappa analysis) to compare the different diagnoses with the gold standard diagnosis. When the kappa value was lower than 0.4 , the accuracy of this kind of diagnosis is poor. When the kappa value is between 0.41 and 0.60 , there are some differences between this type of diagnosis and the gold standard diagnosis. When the kappa value is between 0.61 and 0.80 , the accuracy of this kind of diagnosis is good but can still be improved. When the kappa value is between 0.81 and 1.00 , this kind of diagnosis is very close to the gold standard diagnosis (14). To assess the ability of the skin in situ immune cell detection kit to classify lupus, receiver operating characteristic (ROC) curves were plotted to determine the critical value of each type of lupus. The area under the ROC curves (AUC) was obtained to 


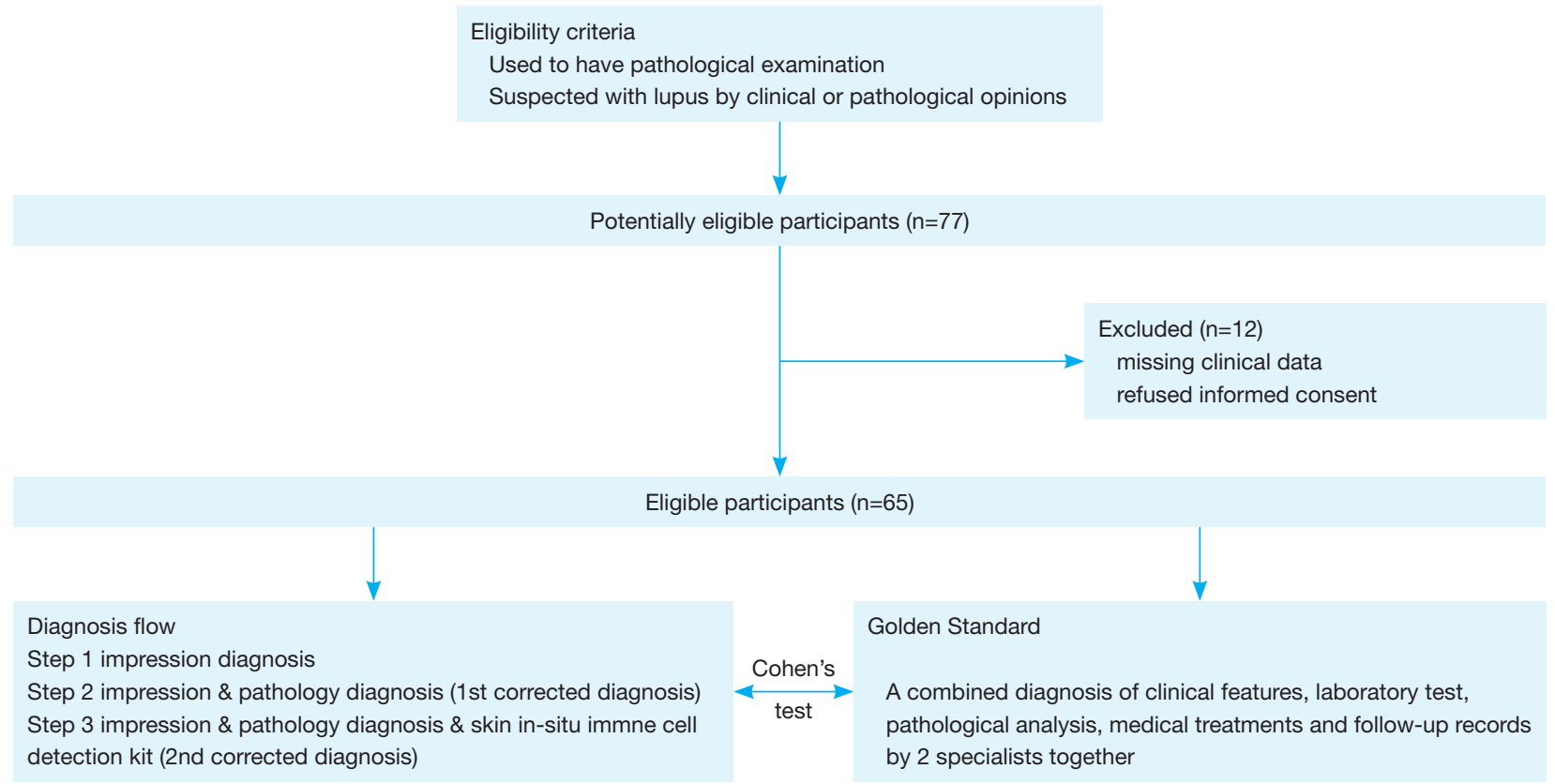

Figure 1 Flow of participants. Patients suspected of having lupus in the clinic were excluded by their failure to complete any one of the clinical tests for lupus, insufficient clinical data or refused informed consent.

reflect the overall accuracy. When the $\mathrm{P}$ value was lower than 0.05 , the data were considered statistically significant.

\section{Results}

\section{The number of $\mathrm{CD}^{+}$and $\mathrm{CD} \mathrm{9}^{+}$cells in situ serves as a referable guide for lupus classification}

After trials on some lupus skin biopsy tissues, we tested the performance of an in situ immune cell detection kit and observed subtle differences in the immune cell distribution and cell numbers (Figure $2 A$ ). We then enrolled patients with DLE $(n=18)$, SCLE $(n=18)$ and ACLE $(n=18)$, all of whom had a rather typical clinical manifestation and a comprehensive diagnosis, and performed an immune-cellcount analysis by comparing the percentage of $\mathrm{CD}^{+}$and $\mathrm{CD} 19^{+}$cell counts over the karyocyte counts among the three groups. The ROC curves (Figure $2 B$ ) and the AUC indicate that comparing $\mathrm{CD} 4^{+}$and $\mathrm{CD} 19^{+}$cell percentages can be an effective way to differentiate subtypes of lupus.

Because of the proliferation of autoreactive $\mathrm{T}$ and $\mathrm{B}$ cells in situ, $\mathrm{CD}^{+}$and $\mathrm{CD}_{19} 9^{+}$cell infiltration in DLE skin lesions is more intensive than that in SCLE and SLE, which makes it easy to rule out DLE from other common lupus subtypes. If the $\mathrm{CD} 4^{+} \%+\mathrm{CD} 19^{+} \%$ of karyocytes in a case was lower than $49.99 \%$, there was a strong possibility that it was not DLE (sensitivity $=83.33 \%$, specificity $=91.67 \%$ ). If it was between $27.25 \%$ and $49.99 \%$, it was likely to be an SCLE case. If it was lower than $27.25 \%$, a diagnosis of ACLE was considered (sensitivity $=88.89 \%$, specificity $=66.67 \%$ ). In addition, the morphology and distribution of the immune cells can be another way to explore the differences among LE subtypes. In DLE, immune cells can form CD19+ cell clusters around cutaneous appendages, while in SLE, immune cells are more scattered around the vessels. These features are noteworthy and have the potential to work as an assistive diagnosis for LE classification.

To determine how much the skin in situ immune cell detection kit can aid in the pathological diagnosis of LE subtype classification statistically, we conducted a Cohen's kappa analysis to compare each set of results with the gold standard diagnosis (a comprehensive assessment of their clinical manifestation, medical history and reports, pathology report and long-term follow-up). The results shown in Table 1 indicate that diagnosis using the skin in situ immune cell detection kit $(\mathrm{k}=0.727)$ shares a higher consistency with the gold standard than the pathology diagnosis $(\mathrm{k}=0.409)$ alone. The problem with the typical pathology diagnosis is that it cannot identify the specific LE subtype, while when using the kit, the total accuracy rate 
A
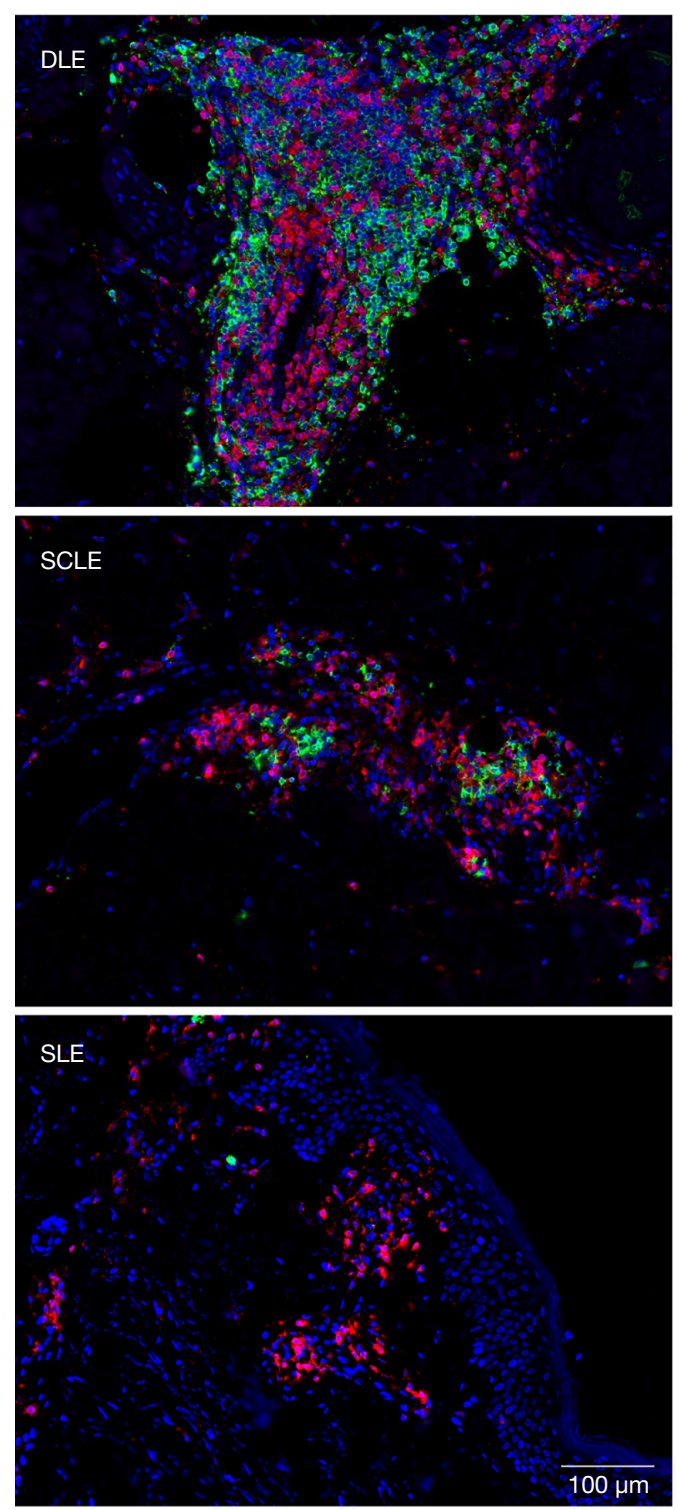

B

ROC curve for distinguishing DLE from SCLE \& SLE by CD4\% + CD19\%

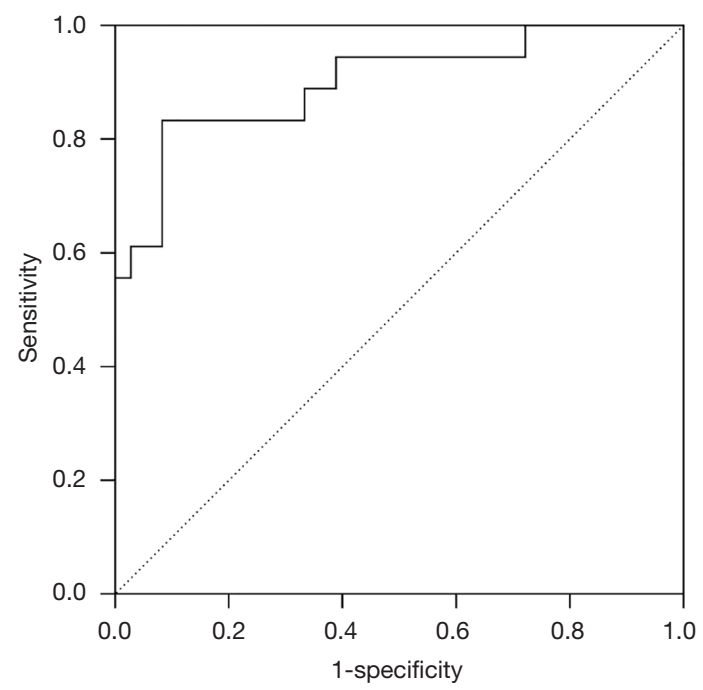

ROC curve for distinguishing SCLE from SLE by CD4\% + CD19\%

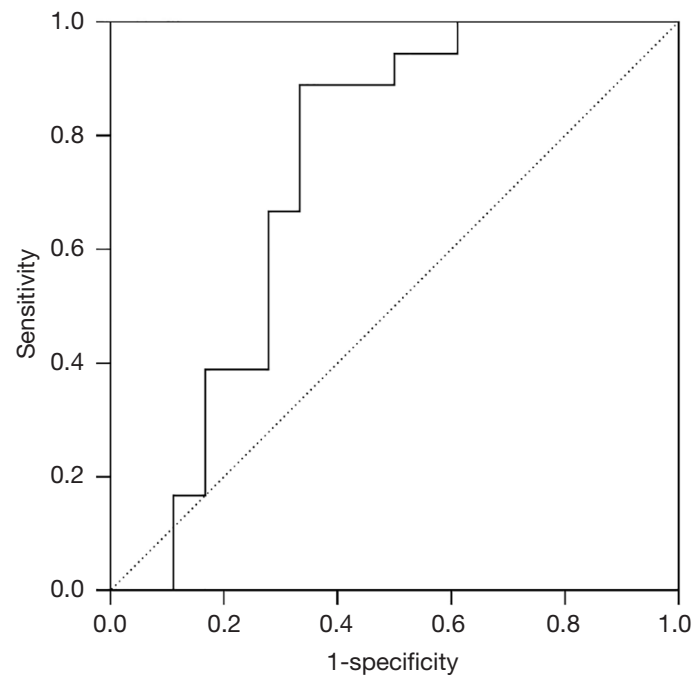

Figure 2 The performance of the skin in situ immune cell detection kit for LE classification. (A) The utility of a skin in situ immune cell detection kit to help identify $\mathrm{CD}^{+}$cells (red) and $\mathrm{CD} 19^{+}$cells (green) and cell nuclei (blue) in skin tissue, and the immune cell distribution and counts show differences among DLE, SCLE, and SLE. (B) Using the percentage of CD4 $4^{+}$and CD19 $9^{+}$cells over karyocytes. Scale bar represents $100 \mu \mathrm{m}$. ROC analysis was performed to distinguish DLE from SCLE and SLE (upper, AUC =0.900) and to distinguish SCLE from SLE (lower, AUC =0.731).

of DLE diagnosis increased from $51.85 \%$ to $81.48 \%$ (DLE from $72.22 \%$ to $94.44 \%$, SCLE from $50.00 \%$ to $77.77 \%$, SLE from $33.33 \%$ to $72.22 \%$ ). In summary, the skin in situ immune cell detection kit can serve as a novel method to improve the pathological LE classification.

The combined application of an in situ immune cell detection kit can assist in differentiating LE from a mix of lupus-like diseases

To further test the skin in situ immune cell detection kit, we randomly recruited 65 patients from 3 different hospitals, and their demographic characteristics are detailed in Table 2. 
Table 1 Skin in-situ immune cell detection kit can aid pathology diagnosis on LE classification

\begin{tabular}{|c|c|c|c|c|c|c|}
\hline Variable & \multicolumn{3}{|c|}{ Comprehensive diagnosis } & Total & Kappa value & $P$ value \\
\hline \multicolumn{7}{|l|}{ Pathology diagnosis } \\
\hline $\mathrm{DLE}^{\mathrm{a}}$ & 13 & 0 & 0 & 13 & 0.409 & 0.000 \\
\hline SCLE $^{\mathrm{b}}$ & 1 & 9 & 0 & 10 & & \\
\hline Undefined LE subtypes $^{d}$ & 4 & 7 & 12 & 23 & & \\
\hline Other types of erythema lesions ${ }^{e}$ & 0 & 1 & 0 & 1 & & \\
\hline Total & 18 & 18 & 18 & 54 & & \\
\hline \multicolumn{7}{|c|}{ A combination of pathology diagnosis \& skin in situ immune cell detection kit } \\
\hline $\mathrm{SLE}^{\mathrm{c}}$ & 0 & 3 & 13 & 16 & & \\
\hline Undefined LE subtypes $^{d}$ & 0 & 0 & 2 & 2 & & \\
\hline Other types of erythema lesions ${ }^{e}$ & 0 & 0 & 0 & 0 & & \\
\hline Total & 18 & 18 & 18 & 54 & & \\
\hline
\end{tabular}

Table 2 Demographic characteristics of 65 patients

\begin{tabular}{lc}
\hline Characteristics & Statistics \\
\hline Sex, No. male/female & $19 / 46$ \\
Age, mean \pm SD years & $42.28 \pm 15.75$ \\
Hematologic involvement, n (\%) & $29.23 \%(19 / 65)$ \\
Renal involvement, $\mathrm{n}(\%)$ & $16.92 \%(11 / 65)$ \\
SLEDAl score $>4, \mathrm{n}(\%)$ & $38.46 \%(25 / 65)$ \\
\hline
\end{tabular}

A total of 65 lesions were comprehensively diagnosed (based on the patients' clinical manifestation, medical history and reports, and pathology report along with long-term followup) by at least two experienced dermatology specialists and were classified into 50 cases of lupus erythematosus (LE), 8 lichen planus (LP), 3 granuloma annulare (GA) and 1 case each vasculitis, erythema annulare centrifugum (EAC), rosacea and lymphoma. We then used this diagnosis as the gold standard and compared the pathology diagnostic accuracy to a combination of pathology and skin in situ immune cell detection kit diagnosis accuracy for distinguishing among these conditions. Our statistical results showed that the accuracy of pathologists in distinguishing LE was $74.00 \%$ (37/50, sensitivity $=74.00 \%$, specificity $=100.00 \%)$. With the kit, the accuracy was enhanced to $98.00 \%$ (49/50, sensitivity $=98.00 \%$, specificity $=93.33 \%$ ). In conclusion, the skin in situ immune cell detection kit can help pathologists identify LE by increasing the sensitivity.

However, in hospitals, the diagnosis of LE must come from an overall consideration. To simulate clinical practice, we successively compared the consistency of each step of our diagnosis processes, including impression diagnosis, pathology diagnosis, the combined consideration of the former two methods, and adding the skin in situ immune cell detection kit to assist the judgments. The accuracy of impression diagnosis was $67.6 \%(44 / 65)$, and the accuracy of pathology diagnosis improved it to $75.3 \%$ (49/65). Combined impression and pathology could improve this accuracy to $92.3 \%$ (60/65), with only 5 cases left without a correct or definite diagnosis. With the help of the skin in situ immune cell detection kit, $60 \%(3 / 5)$ of the remaining cases were correctly diagnosed. To test the statistical significance of our results, we employed Cohen's kappa analysis to compare their consistency to the gold standard (Table 3). 
This demonstrated that the combined utility plus the skin in situ immune cell detection kit has the highest consistency with the gold standard, which provides a new approach to distinguish lupus and similar diseases.

We can see from Table 3 that it is easy for dermatologists to make an incorrect diagnosis at the first impression. With its diverse manifestations, lupus can be mixed with LP, GA, EAC, lymphoma or other related diseases, such as erythema multiforme, solar dermatitis, eczema, etc. Pathologists do not always give a specific judgment toward lupus but rather do not rule it out from among other connective tissue diseases, which also explains the relatively low sensitivity of pathology diagnosis. As clinical dermatologists can consider their opinion based upon the patients' history of disease, clinical manifestations and the pathological diagnosis together, the accuracy of diagnosis can increase. However, some remaining cases need to be subjected to a more powerful approach for further diagnosis, and the use of a skin in situ immune cell detection kit can make a difference in this field and help to further distinguish subtypes of lupus.

\section{A buge step forward for LE classification with a skin in situ immune cell detection kit}

After a long period of follow-up of the patients' progression of their disease and their reactions to medication, 50 lupus cases from Table 4 had their subtypes clearly diagnosed, and the same statistical analysis was conducted for these cases to figure out how far the skin in situ immune cell detection kit can go to identify subtypes of lupus.

As shown in Table 3, these 50 cases were made up of DLE $(n=17)$, SCLE $(n=21)$ and SLE $(n=11)$. In the first step of impression diagnosis, each subtype of LE can be mistaken for other subtypes or be thought of as an uncertain lupus subtype. Only 44\% (22/50) are properly diagnosed, and it is harder to separate SCLE or SLE than DLE. It is worth noting that the lowest consistency for the right subtype diagnosis occurs among pathologists because the pathology diagnosis can be ambiguous or vague about lupus subtypes. However, even though they only diagnosed 34\% (17/50) of cases correctly to the specific to lupus subtypes, there was only 1 case that they diagnosed as other than lupus. Based on the pathology views, it is easy to capture the core feature of LE, but it is quite difficult to further divide them into subtypes. It also seems a burden for clinicians to distinguish LE subtypes based on impression and pathology diagnosis. With an accuracy of only $68 \%(34 / 50)$, many of the cases are classified as uncertain LE subtypes, and difficulty occurs more often when dermatologists need to separate SCLE from DLE or to distinguish SLE from SCLE.

Surprisingly, after the auxiliary diagnosis of the kit, the diagnostic accuracy reached $96 \%(48 / 50)$, as did its consistency with the gold standard $(\mathrm{k}=0.940, \mathrm{P}=0.000)$, which means that the kit can effectively help distinguish most lupus subtypes. In other words, this kit enhanced the LE classification accuracy by $30 \%$ compared to the diagnostic accuracy of impression diagnosis combined with pathological analysis.

In Table 5, we listed the DLE, SCLE and SLE patients separately to compare the performance of the combined diagnosis of impression and pathology with and without the skin in situ immune cell detection kit. From the upper table, the diagnostic accuracy is $58.82 \%(10 / 17)$ without the skin in situ immune cell detection kit. However, with the kit, we can see that all 7 misdiagnosed DLE cases were corrected, showing its great advantages in diagnosing DLE. In the middle table, the kit also displayed its ability to recognize characteristics of SCLE, as its accuracy [58.82\% (20/21)] was much higher than that without the kit [61.90\% (13/21)]. However, in SLE, the diagnostic accuracy with or without the kit seems about the same. A probable explanation for this is that the diagnosis of SLE relies more on a systemic assessment of the patient than pathology staining. When systemic assessment does not meet the standard, it is difficult for a skin in situ immune cell detection kit to provide further evidence.

\section{Discussion}

At present, there are no appropriate classification criteria or auxiliary diagnostic kits for CLE. Clinically, the subtypes of cutaneous lupus are classified according to their cutaneous manifestations, histologic features, laboratory tests, and special pathological analyses, such as lupus band tests (15). Cutaneous histopathology is widely used in clinical practice and is generally regarded as the gold standard for the diagnosis of skin lesions of lupus (16). Our data show that the pathology diagnosis has good specificity but poor sensitivity, which may be because of the lack of specialized clinical knowledge of dermatology and evidence from the laboratory examination when the pathologists make their diagnosis. The lupus band test, is a definitive test to differentiate DLE from SLE, since positive staining can be found in both the lesional and nonlesional skin of 
Page 8 of 12

Table 3 Consistency test for diagnosis of LE to other related diseases

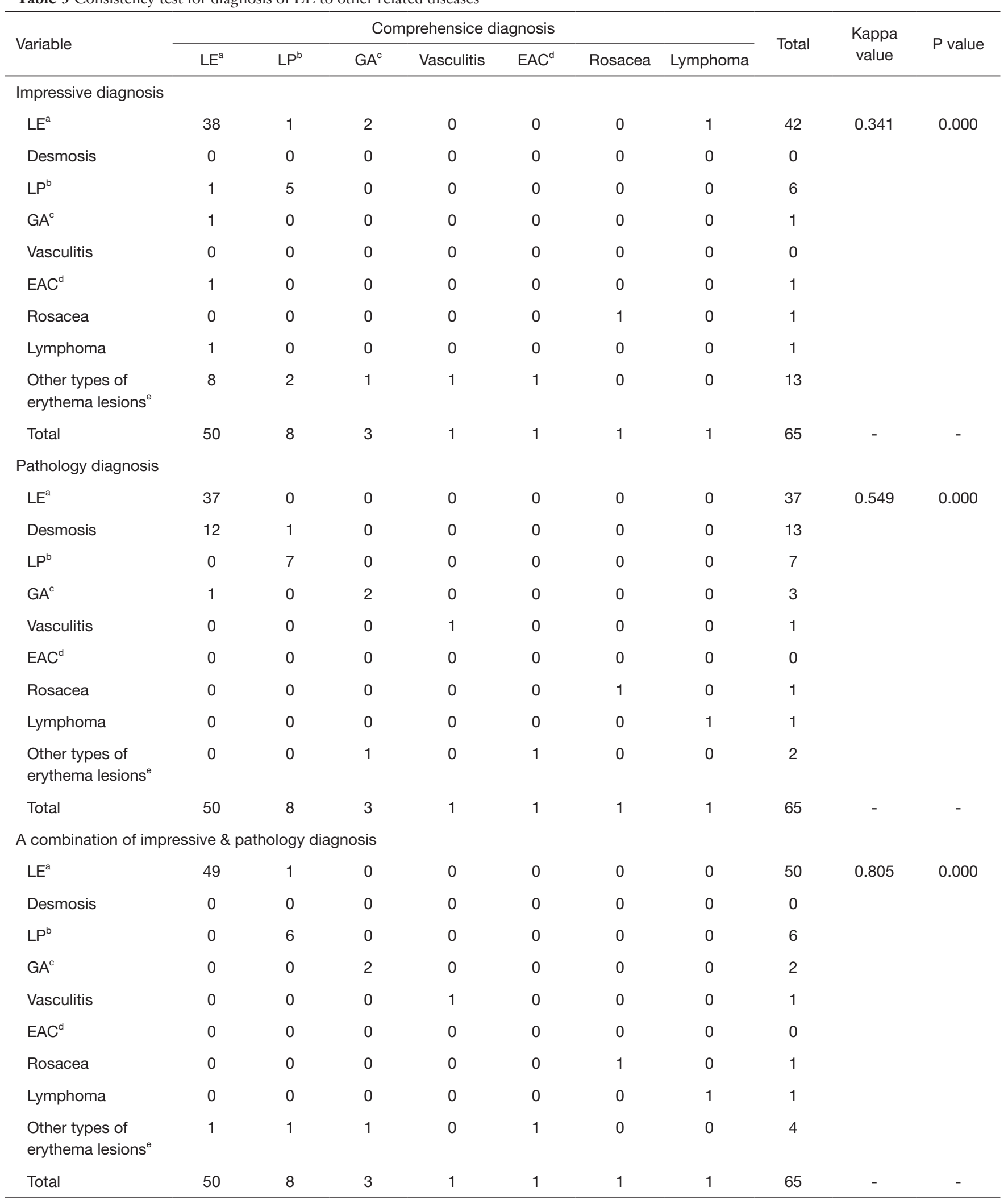

Table 3 (continued) 
Table 3 (continued)

\begin{tabular}{|c|c|c|c|c|c|c|c|c|c|c|}
\hline Variable & \multicolumn{7}{|c|}{ Comprehensice diagnosis } & Total & $\begin{array}{c}\text { Kappa } \\
\text { value }\end{array}$ & $P$ value \\
\hline \multicolumn{11}{|c|}{ A combination of impressive \& pathology diagnosis \& skin in situ immune cell detection kit } \\
\hline $\mathrm{LE}^{\mathrm{a}}$ & 50 & 0 & 0 & 0 & 0 & 0 & 0 & 50 & 0.921 & 0.000 \\
\hline Desmosis & 0 & 0 & 0 & 0 & 0 & 0 & 0 & 0 & & \\
\hline $\mathrm{GA}^{\mathrm{c}}$ & 0 & 0 & 2 & 0 & 0 & 0 & 0 & 2 & & \\
\hline Vasculitis & 0 & 0 & 0 & 1 & 0 & 0 & 0 & 1 & & \\
\hline $\mathrm{EAC}^{\mathrm{d}}$ & 0 & 0 & 0 & 0 & 0 & 0 & 0 & 0 & & \\
\hline Rosacea & 0 & 0 & 0 & 0 & 0 & 1 & 0 & 1 & & \\
\hline Total & 50 & 8 & 3 & 1 & 1 & 1 & 1 & 65 & - & - \\
\hline
\end{tabular}

Table 4 Consistency test for diagnosis of LE subtypes

\begin{tabular}{|c|c|c|c|c|c|c|}
\hline Variable & \multicolumn{3}{|c|}{ Comprehensive diagnosis } & Total & Kappa value & $P$ value \\
\hline \multicolumn{7}{|l|}{ Impressive diagnosis } \\
\hline $\mathrm{DLE}^{\mathrm{a}}$ & 9 & 2 & 0 & 11 & 0.308 & 0.000 \\
\hline $\operatorname{SCLE}^{\mathrm{b}}$ & 2 & 8 & 1 & 11 & & \\
\hline Undefined LE subtypes $^{d}$ & 3 & 3 & 5 & 11 & & \\
\hline Other types of erythema lesions ${ }^{e}$ & 3 & 8 & 1 & 12 & & \\
\hline Total & 17 & 21 & 12 & 50 & & \\
\hline \multicolumn{7}{|l|}{ Pathology diagnosis } \\
\hline $\operatorname{SLE}^{\mathrm{c}}$ & 0 & 0 & 2 & 2 & & \\
\hline Undefined LE subtypes $^{d}$ & 8 & 14 & 10 & 32 & & \\
\hline Other types of erythema lesions ${ }^{e}$ & 0 & 1 & 0 & 1 & & \\
\hline Total & 17 & 21 & 12 & 50 & & \\
\hline
\end{tabular}

Table 4 (continued) 
Table 4 (continued)

\begin{tabular}{|c|c|c|c|c|c|c|}
\hline Variable & \multicolumn{3}{|c|}{ Comprehensive diagnosis } & Total & Kappa value & $P$ value \\
\hline \multicolumn{7}{|c|}{ A combination of impressive \& pathology diagnosis } \\
\hline DLE $^{\mathrm{a}}$ & 10 & 2 & 0 & 12 & 0.577 & 0.000 \\
\hline SCLE $^{\mathrm{b}}$ & 0 & 13 & 0 & 13 & & \\
\hline Undefined LE subtypes $^{d}$ & 7 & 6 & 1 & 14 & & \\
\hline Other types of erythema lesions ${ }^{e}$ & 0 & 0 & 0 & 0 & & \\
\hline Total & 17 & 21 & 12 & 50 & & \\
\hline \multicolumn{7}{|c|}{ A combination of impressive \& pathology diagnosis \& skin in situ immune cell detection kit } \\
\hline $\mathrm{SLE}^{\mathrm{C}}$ & 0 & 0 & 11 & 11 & & \\
\hline Undefined LE subtypes $^{d}$ & 0 & 1 & 1 & 2 & & \\
\hline Other types of erythema lesions ${ }^{e}$ & 0 & 0 & 0 & 0 & & \\
\hline Total & 17 & 21 & 12 & 50 & & \\
\hline
\end{tabular}

SLE, while deposition of immune complexes only occurs in skin damage from DLE. However, SCLE lesions show a similar pattern of deposition of immune complexes. In addition, false positives can also be encountered in rosacea, rheumatoid arthritis, mixed connective tissue disease and many other disorders. Therefore, the lupus bands test is not an appropriate diagnostic and classification method for lupus (17-19).

In this study, we demonstrated that a skin in situ immune cell detection kit can be effectively applied in the diagnosis and classification of cutaneous lupus. In particular, our work highlights that the skin in situ immune cell detection kit is able to aid the diagnosis of skin lesions of DLE, SCLE and SLE, markedly improve the diagnostic accuracy and prevent omissions. In our study, the results showed that, for the first impression of dermatologists, the consistencies with the accurate diagnosis and classification results of lupus were poor $(\mathrm{k}=0.341)$. Similar to our results, previous reports showed that the concordance rate of lupus between the clinical diagnosis of dermatologists and the pathologic diagnosis of pathologists was just $35.12 \%$ (20). When combining impression and pathology results, the consistency is enhanced ( $\mathrm{k}=0.805)$ but not as much as it is improved by the utilization of the kit $(\mathrm{k}=0.921)$. In addition, we proved the advantage of an in situ immune cell detection kit in identifying DLE. SCLE and SLE identification had the highest $(96 \%)$ accuracy $(\mathrm{k}=0.940)$, which indicates a new step forward in the field of precision medicine for the diagnosis and differential diagnosis of lupus and its subtypes.

Regrettably, this study still has several limitations. First, only three hospitals with a total of 65 samples were collected for research. Thus, the disease types might be influenced by hospitalization bias and selection bias. Second, the small sample size may lead to an overinterpretation of the differences among these diagnosis results. In addition, the results of HE staining and the skin in situ immune cell detection kit need to be judged against human experience. The subjective judgments will also introduce some bias. The current study has shown that the ability of the skin in situ immune cell detection kit to distinguish between SCLE and SLE still needs to be improved. In general, larger samples and multicenter research are necessary to verify our results. 
Table 5 Display and comparison of diagnosis with or without in-situ immune cell detection kit

\begin{tabular}{|c|c|c|c|c|}
\hline $\begin{array}{l}\text { A combination of impressive \& } \\
\text { pathology diagnosis }\end{array}$ & \multicolumn{4}{|c|}{ A combination of impressive \& pathology diagnosis \& skin in situ immune cell detection kit } \\
\hline \multicolumn{5}{|l|}{ DLE patients } \\
\hline DLE & 10 & 0 & 0 & 0 \\
\hline SCLE & 0 & 0 & 0 & 0 \\
\hline Undefined LE subtypes & 7 & 0 & 0 & 0 \\
\hline Total & 17 & 0 & 0 & 0 \\
\hline \multicolumn{5}{|l|}{ SCLE patients } \\
\hline DLE & 0 & 2 & 0 & 0 \\
\hline Undefined LE subtypes & 0 & 6 & 0 & 0 \\
\hline Total & 0 & 20 & 0 & 1 \\
\hline \multicolumn{5}{|l|}{ SLE patients } \\
\hline DLE & 0 & 0 & 0 & 0 \\
\hline SCLE & 0 & 0 & 0 & 0 \\
\hline SLE & 0 & 0 & 10 & 0 \\
\hline Undefined LE subtypes & 0 & 0 & 0 & 1 \\
\hline Total & 0 & 0 & 10 & 1 \\
\hline
\end{tabular}

DLE, discoid lupus erythematosus; SCLE, subacute cutaneous lupus erythematosus; SLE, systemic lupus erythematosus.

\section{Acknowledgments}

Funding: This work was supported by the National Natural Science Foundation of China (No. 81830097, No. 81972943, No. 81773332), Key Research and Development Program of Hunan Province (2018XK2304), Hunan Talent Young Investigator (No. 2019RS2012), Hunan Outstanding Young Investigator (No. 2020JJ2055) and CAMS Innovation Fund for Medical Sciences (CIFMS) (2019-I2 M-5-033), and the Nonprofit Central Research Institute Fund of the Chinese Academy of Medical Sciences (No. 2020-RC320003). We would like to thank AJE for their help in polishing our paper.

\section{Footnote}

Reporting Checklist: The authors have completed the STARD reporting checklist. Available at http://dx.doi.org/10.21037/ atm-21-959
Data Sharing Statement: Available at http://dx.doi. org/10.21037/atm-21-959

Conflicts of Interest: All authors have completed the ICMJE uniform disclosure form (available at http://dx.doi. org/10.21037/atm-21-959). The authors have no conflicts of interest to declare.

Ethical Statement: The authors are accountable for all aspects of the work in ensuring that questions related to the accuracy or integrity of any part of the work are appropriately investigated and resolved. The study was conducted in accordance with the Declaration of Helsinki (as revised in 2013). All human studies were approved by the ethics committee of the Second Xiangya Hospital of Central South University (No. 201930044). We obtained written informed consent from all subjects. This study was reviewed and approved by the Ethics Committee and carried out in strict accordance with the approved research protocol. 
Open Access Statement: This is an Open Access article distributed in accordance with the Creative Commons Attribution-NonCommercial-NoDerivs 4.0 International License (CC BY-NC-ND 4.0), which permits the noncommercial replication and distribution of the article with the strict proviso that no changes or edits are made and the original work is properly cited (including links to both the formal publication through the relevant DOI and the license). See: https://creativecommons.org/licenses/by-nc-nd/4.0/.

\section{References}

1. Li Q, Wu H, Liao W, et al. A comprehensive review of immune-mediated dermatopathology in systemic lupus erythematosus. J Autoimmun 2018;93:1-15.

2. Li Q, Wu H, Zhou S, et al. An Update on the Pathogenesis of Skin Damage in Lupus. Curr Rheumatol Rep 2020;22:16.

3. Werth VP. Cutaneous lupus: insights into pathogenesis and disease classification. Bull NYU Hosp Jt Dis 2007;65:200-4.

4. Ribero S, Sciascia S, Borradori L, et al. The Cutaneous Spectrum of Lupus Erythematosus. Clin Rev Allergy Immunol 2017;53:291-305.

5. Panjwani S. Early diagnosis and treatment of discoid lupus erythematosus. J Am Board Fam Med 2009;22:206-13.

6. Chong BF, Werth VP. 33 - Skin Disease in Cutaneous Lupus Erythematosus. In: Wallace DJ, Hahn BH, editors. Dubois' Lupus Erythematosus and Related Syndromes (Ninth Edition). London: Elsevier, 2019:395-406.

7. Haugaard JH, Kofoed K, Gislason G, et al. Association Between Drug Use and Subsequent Diagnosis of Lupus Erythematosus. JAMA Dermatol 2020;156:1199-207.

8. Guicciardi F, Atzori L, Marzano AV, et al. Are there distinct clinical and pathological features distinguishing idiopathic from drug-induced subacute cutaneous lupus

Cite this article as: Li Q, Chen K, Deng Y, Liu X, Ma G, Zeng J, Lu L, Zhao M, Wu H, Lu Q. A skin in situ immune cell detection kit for the diagnosis and classification of cutaneous lupus erythematosus. Ann Transl Med 2021;9(13):1062. doi: 10.21037/atm-21-959 erythematosus? A European retrospective multicenter study. J Am Acad Dermatol 2019;81:403-11.

9. Papachristos DA, Oon S, Hanly JG, et al. Management of inflammatory neurologic and psychiatric manifestations of systemic lupus erythematosus: A systematic review. Semin Arthritis Rheum 2021;51:49-71.

10. Tsokos GC. Autoimmunity and organ damage in systemic lupus erythematosus. Nat Immunol 2020;21:605-14.

11. Bockle BC, Sepp NT. Smoking is highly associated with discoid lupus erythematosus and lupus erythematosus tumidus: analysis of 405 patients. Lupus 2015;24:669-74.

12. Kiriakidou M, Ching CL. Systemic Lupus Erythematosus. Ann Intern Med 2020;172:ITC81-ITC96.

13. Karaaslan S, Tomayko MM. A Niche for Plasma Cells: The Skin. J Invest Dermatol 2019;139:2411-4.

14. Landis JR, Koch GG. The measurement of observer agreement for categorical data. Biometrics 1977;33:159-74.

15. Okon LG, Werth VP. Cutaneous lupus erythematosus: diagnosis and treatment. Best Pract Res Clin Rheumatol 2013;27:391-404.

16. Tani C, Carli L, Vagnani S, et al. The diagnosis and classification of mixed connective tissue disease. J Autoimmun 2014;48-49:46-9.

17. Lahita RG. Chapter 32 - Systemic Lupus Erythematosus. In: Rose NR, Mackay IR, editors. The Autoimmune Diseases (Fifth Edition). Boston: Academic Press; 2014:451-61.

18. Gangaram HB, Kong NC, Phang KS, et al. Lupus band test in systemic lupus erythematosus. Med J Malaysia 2004;59:638-48.

19. Kulthanan K, Roongphiboolsopit P, Chanjanakijskul S, et al. Chronic discoid lupus erythematosus in Thailand: direct immunofluorescence study. Int J Dermatol 1996;35:711-4.

20. Analysis of clinical and pathological diagnoses of 29987 skin biopsy samples in Peking Union Medical College Hospital. Chinese Journal of Dermatology 2020;53. 